\title{
Assistência de enfermagem ao paciente oncológico submetido à anestesia
}

\author{
Nursing care for cancer patients undergoing anesthesia \\ Atención de enfermería para pacientes con cáncer sometidos a anestesia
}

Jonas Magno dos Santos Cesário ORCID: https://orcid.org/0000-0003-1785-3555 Faculdade Unyleya ${ }^{1}$, Brasil

E-mail: prof.jonasmagno@unyleya.edu.br

Victor Hugo de Paula Flauzino ORCID: https://orcid.org/0000-0001-5156-0030 Faculdade Unyleya ${ }^{2}$, Brasil

E-mail: prof.victorflauzino@unyleya.edu.br

Luana de Oliveira Hernandes

ORCID: http://orcid.org/0000-0001-8828-7146 Associação Brasileira de Enfermeiros cientistas, Brasil E-mail: luanaoliveira2306@gmail.com

Daiana Moreira Gomes

ORCID: https://orcid.org/0000-0001-9387-0619 Associação Brasileira de Enfermeiros cientistas, Brasil E-mail: daigomes_87@hotmail.com

Priscila Gramata da Silva Vitorino

ORCID: https://orcid.org/0000-0002-1201-6945 Associação Brasileira de Enfermeiros cientistas, Brasil E-mail: Prigramaenf@yahoo.com.br

\begin{abstract}
Resumo
O objetivo deste trabalho foi descrever os riscos associados à anestesia no paciente oncológico e os cuidados de enfermagem durante anestesia no paciente oncológico. A pesquisa é uma revisão bibliográfica de abordagem descritiva e qualitativa. Foi realizada nos Bancos de dados do Google Acadêmico, BVS (biblioteca virtual em saúde) e SciELO (ScientificElectronic Library Online), com a seguinte pergunta de pesquisa: quais os cuidados de enfermagem ao paciente oncológico submetido à anestesia? Foram inclusos os artigos acadêmicos publicados entre 2012 á 2020 , na língua portuguesa, disponíveis de forma gratuitos. Foram excluídos os artigos inferiores a 2012, resumos, periódicos que não contemplavam nenhum dos objetivos, que não respondessem à pergunta de pesquisa e artigos repetidos encontrados nas bases de dados citadas acima, no qual resultou em uma amostra final de 35 artigos. As complicações anestesia geral em pacientes oncológicos são o desenvolvimento de paralisia das cordas vocais após a intubação, o tempo prolongado de jejum, edema pulmonar, atelectasia, pneumonia, obstrução da VAS (vias aéreas superiores), broncoespasmo, edema de VAS, bronquite e exacerbar DPOC (Doença Pulmonar Obstrutiva Crônica), e os anestésicos utilizados podem gerar complicações como: fraqueza da musculatura faríngea, depressão respiratória, pruridos, náuseas, vômitos, retenção de urina, diminuição da motilidade gastrointestinal e obstrução intestinal paralítica. A atuação do enfermeiro nos procedimentos anestésicos é fundamental para promover um ambiente seguro e propicio para a anestesia e realizar uma assistência de enfermagem segura para ter um procedimento cirúrgico tranquilo.
\end{abstract}

Palavras-chave: Cuidados de enfermagem; Anestesia; Oncologia cirúrgica.

\begin{abstract}
The objective of this study was to describe the risks associated with anesthesia in cancer patients and nursing care during anesthesia in cancer patients. The research is a bibliographic review of descriptive and qualitative approach. It was carried out in the Databases of Google Acadêmico, BVS (biblioteca virtual em saúde) e SciELO (ScientificElectronic Library Online), with the following research question: what nursing care for cancer patients submitted to anesthesia? Academic articles published between 2012 and 2020 were included, available free of charge. Articles lower than 2012, abstracts, journals that did not contemplate any of the objectives, that did not answer the research question and repeated articles found in the databases mentioned above, which resulted in a final sample of 35 articles, were excluded. Complications general anesthesia in cancer patients are the development of vocal cord paralysis after intubation, prolonged fasting time, pulmonary edema, atelectasis, pneumonia, VAS obstruction (upper airways), bronchospasm, VAS edema, bronchitis and exacerbating COPD (Chronic Obstructive Pulmonary Disease),
\end{abstract}

\footnotetext{
${ }^{1}$ Departamento de Enfermagem, Programa de Pós-Graduação em enfermagem em recuperação pós-anestésica.
}

${ }^{2}$ Departamento de Enfermagem, Programa de Pós-Graduação em enfermagem em recuperação pós-anestésica. 
and the anesthetics used can generate complications such as: weakness of the pharyngeal musculature, respiratory depression, pruritus, nausea, vomiting, urine retention, decreased gastrointestinal motility, and paralytic bowel obstruction. The role of nurses in anesthetic procedures is fundamental to promote a safe and favorable environment for anesthesia and to perform safe nursing care to have a quiet surgical procedure.

Keywords: Nursing care; Anesthesia; Surgical oncology.

\section{Resumen}

El objetivo de este estudio era describir los riesgos asociados con la anestesia en pacientes con cáncer y la atención de enfermería durante la anestesia en pacientes con cáncer. La investigación es una revisión bibliográfica del enfoque descriptivo y cualitativo. Se llevó a cabo en las bases de datos de la Google Acadêmico, BVS (biblioteca virtual em saúde) e SciELO (ScientificElectronic Library Online), con la siguiente pregunta de investigación: ¿qué cuidado de enfermería se lleva a los pacientes con cáncer sometidos a anestesia? Se incluyeron artículos académicos publicados entre 2012 y 2020, disponibles de forma gratuita. Se excluyeron artículos inferiores a 2012, resúmenes, revistas que no contemplaban ninguno de los objetivos, que no respondían a la pregunta de investigación y artículos repetidos encontrados en las bases de datos mencionadas anteriormente, lo que dio lugar a una muestra final de 35 artículos. Las complicaciones generales de la anestesia en pacientes con cáncer son el desarrollo de parálisis de las cuerdas vocales después de la intubación, El tiempo prolongado de ayuno, el edema pulmonar, la atelectasia, la neumonía, la obstrucción del VAS (vías respiratorias superiores), el broncoespasmo, el edema vas, la bronquitis y la EPOC exacerbante (enfermedad pulmonar obstructiva crónica) y los anestésicos utilizados pueden generar complicaciones tales como: debilidad de los músculos faríngeos, depresión respiratoria, picazón, náuseas, vómitos, retención de orina, disminución de la motilidad gastrointestinal y obstrucción intestinal paralítica. El papel de las enfermeras en los procedimientos anestésicos es fundamental para promover un ambiente seguro y favorable para la anestesia y para realizar una atención de enfermería segura para someterse a un procedimiento quirúrgico silencioso.

Palabras clave: Cuidados de enfermería, Anestesia; Oncología quirúrgica.

\section{Introdução}

A anestesia do paciente oncológico é desafiadora para os profissionais de saúde, pois apresenta várias comorbidades como a dor crônica de difícil controle, uso de quimioterápicos ou radioterápicos, levando o paciente a ser resistente a diversos anestésicos e analgésicos opioides, no qual torna o momento de indução anestésico complexo (Lima et al., 2019).

Durante a anestesia, a escolha de drogas ou técnicas de anestésicas específicas devem considerar as condições cirúrgicas intraoperatórias adequadas e ajustáveis e, então, avalia-se a rápida recuperação com o mínimo de efeitos colaterais e retornar rapidamente às atividades psicomotoras normais. Isto requer do enfermeiro tenha o conhecimento técnico e prático para prestar assistência de enfermagem segura durante a indução anestésica (Garcia et al., 2020)

O enfermeiro é o profissional habilitado para gerenciar todas as necessidades que circundam a anestesia realizada durante as cirurgias de pacientes oncológicos, com o objetivo a promoção, recuperação, cuidado individualizado, humanizado, assim como prevenir possíveis complicações pós-operatórias ao paciente. Além disso, proporcionar para pacientes e seus familiares um cuidado indireto. O objetivo é oferecer uma assistência de enfermagem com qualidade que visa promover resultados positivos (Sobral et al., 2020)

O enfermeiro necessita vasto conhecimento acerca do período perioperatório, especialmente no momento anestésicocirúrgico, pois é um momento em que a privacidade do paciente é invadida, se fazendo necessária singularidade do atendimento e humanização dos enfermeiros. A equipe de enfermagem desempenha papel único em todo o período perioperatório, tendo o enfermeiro como o agente que vai aplicar todo o seu conhecimento técnico científico com a finalidade de oferecer um cuidado sistematizado e efetivo. Não basta apenas conhecer determinadas técnicas, o paciente precisa ser avaliado como um todo, e é nesse momento que a equipe de enfermagem pode e deve mostrar todo o seu diferencial enquanto classe profissional (Santos et al., 2017).

O paciente oncológico requer cuidados especiais, principalmente quando submetido a um procedimento cirúrgico, pois o uso da anestesia é indispensável para que o procedimento ocorra. O tratamento cirúrgico em pacientes oncológicos é geralmente a primeira escolha. Diante disso, o uso de diferente técnicas e tipos de anestésicos estão sendo estudados, como meio de descobrir quais os desfechos que esses fármacos e técnicas podem fazer nesses tipos de pacientes, sendo possível que 
esses procedimentos reduzam as respostas negativas que possam surgir no período operatório e pós-operatório, por meio disto surgiu a seguinte pergunta de pesquisa, quais os cuidados de enfermagem ao paciente oncológico submetido à anestesia?

Os cuidados de enfermagem ao paciente oncológico durante a indução anestésica têm como objetivo de promover, recuperar e prevenir o estado de saúde do paciente. Esse processo se dá através do uso da Sistematização da Assistência de enfermagem como o objetivo de padronização e organização do processo de enfermagem. O cuidado de enfermagem primordial é manter os parâmetros de sinais vitais estáveis, equilíbrio hemodinâmico e controle da dor, detectar e prevenir eventos adversos, minimizando qualquer complicação. Avaliado pela equipe de enfermagem no intraoperatório e estão sempre disponíveis para prestar assistência, atender o indivíduo que apresenta dor, limitações, mantendo sempre a humanização e clareza nas ações tomadas na assistência para recuperação do indivíduo, com isto o trabalho tem o objetivo de descrever os riscos associados à anestesia no paciente oncológico e os cuidados de enfermagem durante anestesia no paciente oncológico.

\section{Metodologia}

O estudo foi realizado por meio de uma revisão bibliográfica de abordagem descritiva e qualitativa. Segundo Cesário, Flauzino e Mejia (2020), fundamenta-se com base em material que já foram construídos, o que incluí artigos científicos publicados em periódicos acadêmicos. Na primeira etapa, buscou-se reunir evidências para responder a seguinte pergunta de pesquisa: Quais os cuidados de enfermagem ao paciente oncológico submetido à anestesia?

No DeCS (Descritores em Ciências da Saúde), encontrou-se os seguintes descritores: Cuidados de enfermagem, anestesia e oncologia cirúrgica. Os bancos de dados utilizados foram o Google Acadêmico, BVS (biblioteca virtual em saúde) e SciELO (ScientificElectronic Library Online). No Google Acadêmico utilizou-se cada um dos descritores entre aspas ("'). Na BVS (biblioteca virtual em saúde), foi utilizado a opção pesquisa avançada, selecionada as bases da BDENF (Banco de Dados em Enfermagem), LILACS (Literatura Latino-Americana e do Caribe em Ciências da Saúde) e o operador lógico booleano "OR e "AND". Na SciELO (ScientificElectronic Library Online), foi utilizada a opção pesquisa avançada e o operador lógico booleano "OR" e "AND".

Estabeleceu-se como critérios de inclusão, artigos acadêmicos publicados entre 2015 a 2020, na língua portuguesa, disponíveis de forma gratuita e nos bancos de dados já mencionados, que respondessem à pergunta de pesquisa. Excluíram-se artigos repetidos encontrados nas bases de dados, resumos, artigos inferiores a 2012 e artigos que não respondiam o problema da pesquisa. A coleta dos dados foi realizada no mês de março, por 5 pesquisadores de forma independente. Os resultados das buscas pelos dados e do número final de publicações que irão compor a revisão serão apresentados por meio de PRISMA na forma de fluxograma. 
Figura 1. Fluxograma de Prisma.

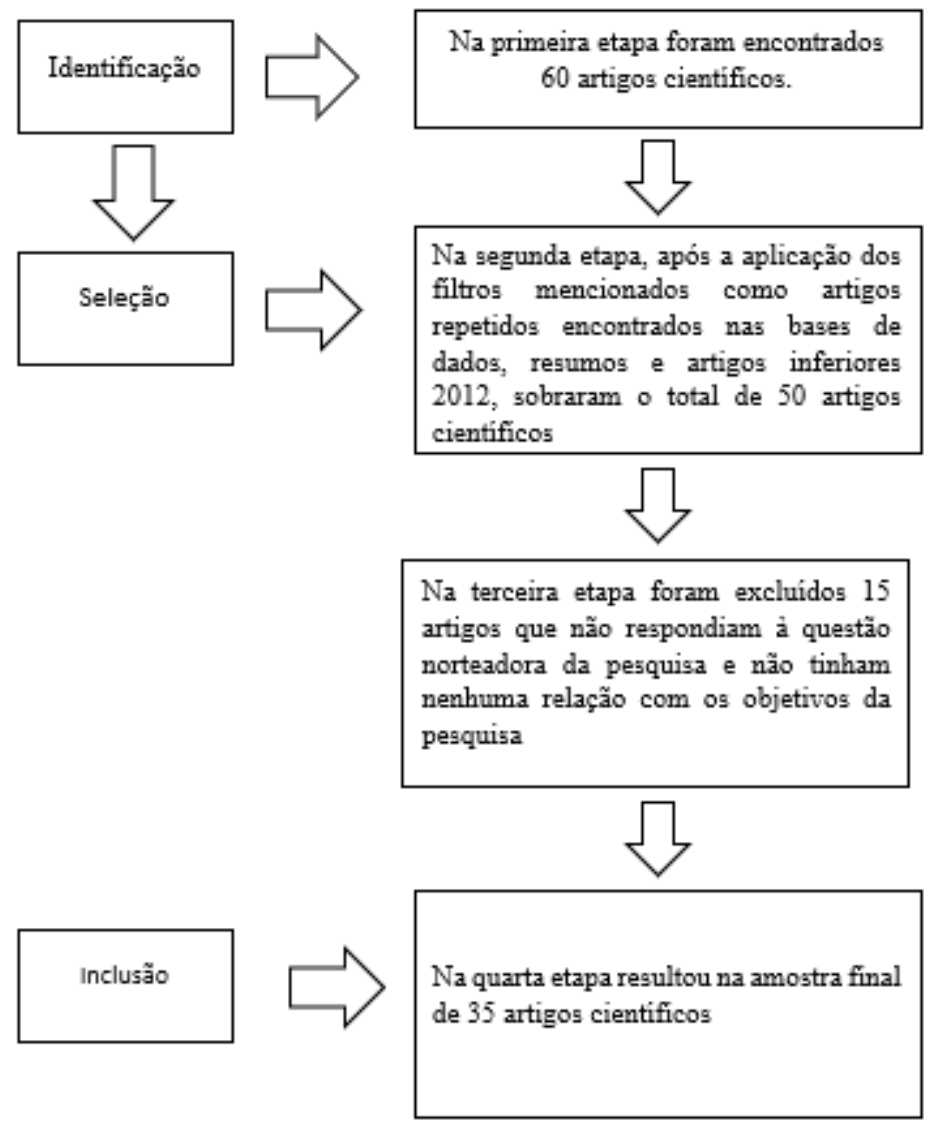

Fonte: Autores (2021).

\section{Resultados}

Na Tabela 1 é possível visualizar a distribuição inicial dos artigos científicos encontrados nas bases de dados da BVS, ScIELO e Google Acadêmico.

Tabela 1. Artigos inclusos neste estudo.

\begin{tabular}{|c|c|c|c|c|c|c|}
\hline \multicolumn{2}{|c|}{ BVS } & \multicolumn{2}{c|}{ ScIELO } & Google Acadêmico & Amostra final \\
\hline Total & 15 & Total & 20 & Total & 25 & \multirow{2}{*}{35 artigos } \\
\hline Excluídos & 06 & Excluídos & 12 & Excluídos & 07 & \\
\hline Incluídos & 09 & Incluídos & 8 & Incluídos & 18 & \\
\hline
\end{tabular}

Fonte: Autores (2021).

Durante a realização da pesquisa os resultados encontrados foram divididos em duas categorias A e B de acordo com a sua temática, a categoria A é composta por riscos associados à anestesia no paciente oncológico e na categoria B cuidados de enfermagem durante anestesia no paciente oncológico.

No Quadro 1, os artigos de revisão da literatura encontrados e utilizados na Categoria A contém as seguintes variáveis: autor, título, objetivo e Periódico. 
Quadro 1. Artigos incluídos na categoria temática A.

\begin{tabular}{|c|c|c|c|}
\hline Autor/ano & Título & Objetivos & Periódico \\
\hline Brasil et al., 2018 & $\begin{array}{l}\text { Métodos anestésicos } \\
\text { intervencionistas no tratamento da } \\
\text { dor oncológica }\end{array}$ & $\begin{array}{l}\text { Descrever os métodos anestésicos } \\
\text { intervencionistas utilizados no } \\
\text { tratamento da dor oncológica. }\end{array}$ & $\begin{array}{l}\text { Acta méd. (Porto } \\
\text { Alegre) }\end{array}$ \\
\hline Nazal et al., 2018 & $\begin{array}{l}\text { Paralisia de cordas vocais após } \\
\text { intubação endotraqueal: uma } \\
\text { complicação incomum da anestesia } \\
\text { geral }\end{array}$ & $\begin{array}{l}\text { Analisar os fatores de risco associados } \\
\text { ao aumento do risco de paralisia das } \\
\text { cordas vocais descritos na literatura. }\end{array}$ & $\begin{array}{l}\text { Revista Brasileira } \\
\text { de Anestesiologia }\end{array}$ \\
\hline $\begin{array}{l}\text { Valdés, Garcia \& } \\
\text { Martínez, } 2019\end{array}$ & $\begin{array}{l}\text { Perioperatorio y recurrencia } \\
\text { oncológica: reto actual en la } \\
\text { práctica anestésica }\end{array}$ & $\begin{array}{l}\text { Descrever os principais fatores } \\
\text { relacionados à anestesia que, durante o } \\
\text { período perioperatório, influenciam a } \\
\text { recorrência oncológica. }\end{array}$ & $\begin{array}{l}\text { Jornal Cubano de } \\
\text { Anestesiologia e } \\
\text { Reanimação }\end{array}$ \\
\hline Silva et al., 2020 & $\begin{array}{l}\text { Pós-operatório de paciente } \\
\text { oncológico em jejum prolongado: } \\
\text { diagnósticos e intervenções de } \\
\text { enfermagem }\end{array}$ & $\begin{array}{l}\text { Identificar os principais diagnósticos de } \\
\text { enfermagem e suas respectivas } \\
\text { intervenções em uma população de } \\
\text { pacientes oncológicos submetidos a } \\
\text { jejum prolongado no pós-operatório. }\end{array}$ & $\begin{array}{l}\text { Revista } \\
\text { Enfermagem } \\
\text { UERJ }\end{array}$ \\
\hline Munhoz et al., 2020 & $\begin{array}{l}\text { Uso de anestesia tumescente em } \\
\text { mastectomia radical unilateral- } \\
\text { relato de caso }\end{array}$ & $\begin{array}{l}\text { Relatar o uso da anestesia local } \\
\text { tumescente em mastectomia radical } \\
\text { unilateral. }\end{array}$ & SIEPE \\
\hline Garcia et al., 2020 & $\begin{array}{l}\text { Ventajas de la anestesia } \\
\text { intravenosa libre de opioides en } \\
\text { cirugía ambulatoria oncológica de } \\
\text { mama }\end{array}$ & $\begin{array}{l}\text { Avaliar o comportamento } \\
\text { hemodinâmico e a recuperação } \\
\text { anestésica em pacientes submetidas a } \\
\text { cirurgia ambulatorial de câncer de } \\
\text { mama. }\end{array}$ & $\begin{array}{l}\text { Revista de Ciências } \\
\text { Médicas de Pinar } \\
\text { del Río }\end{array}$ \\
\hline Antunes et al., 2017 & $\begin{array}{l}\text { Recorrência do câncer: Anestesia é } \\
\text { realmente vilã? }\end{array}$ & $\begin{array}{l}\text { Analisaram-se os principais fatores Peri } \\
\text { operatórios com potencial de } \\
\text { influenciar o sistema imune, } \\
\text { investigando ainda sua associação com } \\
\text { a recidiva tumoral em pacientes } \\
\text { oncológicos. }\end{array}$ & $\begin{array}{l}\text { Rev. Bras. Cir. } \\
\text { Cabeça Pescoço. }\end{array}$ \\
\hline Paiva et al., 2015 & $\begin{array}{l}\text { Complicações respiratórias em } \\
\text { cirurgias oncológicas de grande } \\
\text { porte em cabeça e pescoço: } \\
\text { Revisão de literatura. }\end{array}$ & $\begin{array}{l}\text { Estabelecer a relação, descrita na } \\
\text { literatura, entre cirurgias de grande } \\
\text { porte em cabeça e pescoço e } \\
\text { complicações respiratórias. }\end{array}$ & $\begin{array}{l}\text { Revisa Brasileira } \\
\text { de Anestesiologia. }\end{array}$ \\
\hline Yamada et al., 2019 & $\begin{array}{l}\text { Desenvolvimento de um modelo } \\
\text { preditivo multivariado para náusea } \\
\text { e vômito no pós-operatório de } \\
\text { cirurgia oncológica em adultos. }\end{array}$ & $\begin{array}{l}\text { A previsão do risco de náusea e vômito } \\
\text { no pós-operatório é a base para a } \\
\text { decisão da profilaxia. }\end{array}$ & $\begin{array}{l}\text { Revisa Brasileira } \\
\text { de Anestesiologia. }\end{array}$ \\
\hline
\end{tabular}




\begin{tabular}{|c|c|c|c|}
\hline $\begin{array}{l}\text { Lin, Gill \& Kumar, } \\
2019\end{array}$ & $\begin{array}{l}\text { Bloqueio bilateral do plano eretor } \\
\text { da espinha torácica inferior em } \\
\text { cirurgia oncológica ginecológica } \\
\text { aberta por via abdominal: série de } \\
\text { casos. }\end{array}$ & $\begin{array}{l}\text { O bloqueio do plano do músculo eretor } \\
\text { da espinha é um novo bloqueio } \\
\text { troncular analgésico popularizado } \\
\text { devido à sua facilidade de aplicação e } \\
\text { segurança percebida. }\end{array}$ & $\begin{array}{l}\text { Revista Sociedade } \\
\text { Portuguesa } \\
\text { Anestesiologia. }\end{array}$ \\
\hline Alves \& Faria, 2014 & $\begin{array}{l}\text { Anestesia e recidiva oncológica - } \\
\text { Será tempo de agir? }\end{array}$ & $\begin{array}{l}\text { Influência da técnica anestésica } \\
\text { utilizada na cirurgia oncológica sobre a } \\
\text { função imune no perioperatório e na } \\
\text { recidiva de câncer. }\end{array}$ & $\begin{array}{l}\text { Revista Mexicana } \\
\text { de Anestesiologia. }\end{array}$ \\
\hline $\begin{array}{l}\text { Guzmán \& López, } \\
2019\end{array}$ & $\begin{array}{l}\text { Analgesia perioperatoria en el } \\
\text { paciente oncológico con uso } \\
\text { crónico de opioides. }\end{array}$ & $\begin{array}{l}\text { Avaliação pré-operatória é identificar } \\
\text { pacientes em risco de complicações e } \\
\text { comorbidades para otimizar sua } \\
\text { aptidão, e é um momento apropriado } \\
\text { para avaliar e determinar os métodos } \\
\text { que serão usados para controlar dor. }\end{array}$ & $\begin{array}{l}\text { Revista Brasileira } \\
\text { de Cirurgia Cabeça } \\
\text { Pescoço. }\end{array}$ \\
\hline $\begin{array}{l}\text { Muñoz \& Escobar, } \\
2019\end{array}$ & $\begin{array}{l}\text { Anestesia total intravenosa em } \\
\text { cirurgia de câncer de mama }\end{array}$ & $\begin{array}{l}\text { Descrever os resultados da anestesia } \\
\text { venosa total em cirurgia de câncer de } \\
\text { mama. }\end{array}$ & $\begin{array}{l}\text { Revista Cubana de } \\
\text { Anestesiología y } \\
\text { Reanimación }\end{array}$ \\
\hline $\begin{array}{l}\text { Portella \& Stumm, } \\
2016\end{array}$ & $\begin{array}{l}\text { Intensidade da dor de pacientes em } \\
\text { pós-operatório de cirurgia } \\
\text { oncológica }\end{array}$ & $\begin{array}{l}\text { Avaliar a dor de pacientes oncológicos, } \\
\text { submetidos a cirurgia, assistidos em } \\
\text { uma URPA e compará-la em três } \\
\text { momentos distintos: ao chegar na } \\
\text { URPA, } 1 \text { hora após a e por ocasião da } \\
\text { alta da respectiva unidade. }\end{array}$ & $\begin{array}{l}\text { Salão do } \\
\text { Conhecimento, } \\
\text { Universidade } \\
\text { Regional de Unujuí }\end{array}$ \\
\hline $\begin{array}{l}\text { Pinto \& Grigoletti, } \\
2015\end{array}$ & $\begin{array}{l}\text { Abreviação do jejum entre } \\
\text { pacientes submetidos à cirurgia } \\
\text { oncológica: revisão sistemática }\end{array}$ & $\begin{array}{l}\text { Conduzir uma revisão sistemática a } \\
\text { partir de ensaios clínicos randomizados } \\
\text { controlados para detectar se a } \\
\text { abreviação do jejum traz benefícios } \\
\text { para indivíduos submetidos à cirurgia } \\
\text { oncológica comparativamente aos } \\
\text { protocolos de jejum tradicionais. }\end{array}$ & $\begin{array}{ll}\text { ABCD } & \text { Arquivo } \\
\text { Brasileiro } & \text { Cirurgia } \\
\text { Digestiva } & \end{array}$ \\
\hline $\begin{array}{l}\text { Rangel, Simões \& } \\
\text { Auler, } 2020\end{array}$ & $\begin{array}{l}\text { Anestesia no paciente oncológico: } \\
\text { as técnicas e agentes anestésicos } \\
\text { podem influenciar o desfecho } \\
\text { destes pacientes? Uma revisão } \\
\text { narrativa }\end{array}$ & $\begin{array}{l}\text { Discutir o papel específico de diversos } \\
\text { agentes anestésicos, principalmente } \\
\text { agentes hipnóticos endovenosos, } \\
\text { inalatórios e opioides } \\
\text { Abordar as possíveis diferenças entre as } \\
\text { técnicas anestésicas. }\end{array}$ & Revista Médica \\
\hline $\begin{array}{l}\text { Kearsley, Egan \& } \\
\text { Mccaul, } 2018\end{array}$ & $\begin{array}{lcr}\text { Anestesia } & \text { para } & \text { cirurgia } \\
\text { citorredutora } & (\mathrm{CRS}) & \text { com } \\
\text { quimioterapia } & \text { intraperitoneal } \\
\text { hipertérmica } & \text { (HIPEC) }\end{array}$ & $\begin{array}{lc}\text { Descrever o uso da } & \text { anestesia para } \\
\text { cirurgia citorredutora } & \text { (CRS) com } \\
\text { quimioterapia } & \text { intraperitoneal } \\
\text { hipertérmica (HIPEC) } & \end{array}$ & Anaesthesia \\
\hline
\end{tabular}


No Quadro 2 os artigos de revisão da literatura encontrados e utilizados na categoria B e contém as seguintes variáveis: autor, título, objetivo e Periódico.

Quadro 2. Artigos incluídos na categoria temática B.

\begin{tabular}{|c|c|c|c|}
\hline Autor/ano & Título & Objetivos & Periódico \\
\hline Santos et al., 2017 & $\begin{array}{l}\text { A importância da Assistência de } \\
\text { Enfermagem na Sala de } \\
\text { Recuperação Pós-Anestésica: } \\
\text { Visão dos Monitores em } \\
\text { Enfermagem Cirúrgica. }\end{array}$ & $\begin{array}{l}\text { Identificar a importância da } \\
\text { assistência de enfermagem prestada } \\
\text { ao paciente na Sala de Recuperação } \\
\text { Pós-Anestésica (SRPA). }\end{array}$ & $\begin{array}{l}\text { Universidade } \\
\text { Tiradentes: } \\
\text { International } \\
\text { Nursing Congress }\end{array}$ \\
\hline $\begin{array}{l}\text { Santana, Souza \& } \\
\text { Viana, } 2018\end{array}$ & $\begin{array}{l}\text { Análise das ações de enfermagem } \\
\text { nas fases Cirúrgicas da } \\
\text { mastectomia: uma revisão } \\
\text { sistemática }\end{array}$ & $\begin{array}{l}\text { Analisar o papel da enfermagem nas } \\
\text { fases cirúrgicas da mastectomia, } \\
\text { identificando os fatores } \\
\text { que influenciam no cuidado do } \\
\text { enfermeiro à mulher mastectomizada. }\end{array}$ & $\begin{array}{lr}\text { Revista } & \text { Brasileira } \\
\text { de } & \text { Inovação } \\
\text { Tecnológica } \quad \text { em } \\
\text { Saúde }\end{array}$ \\
\hline Torres et al., 2020 & $\begin{array}{l}\text { Assistência de enfermagem } \\
\text { perioperatória ao paciente } \\
\text { submetido a laparotomia }\end{array}$ & $\begin{array}{l}\text { Descrever a } \begin{array}{l}\text { Sistematização da } \\
\text { Assistência }\end{array} \text { de Enfermagem } \\
\text { Perioperatória (SAEP) ao paciente } \\
\text { vítima de perfuração por PAF subme- } \\
\text { tido a cirurgia de laparotomia. }\end{array}$ & $\begin{array}{l}\text { Enfermagem: } \\
\text { Inovação, } \\
\text { Tecnologia } \quad \text { e } \\
\text { Educação em Saúde }\end{array}$ \\
\hline Lemos \& Peniche, 2016 & $\begin{array}{l}\text { Assistência de enfermagem no } \\
\text { procedimento anestésico: revisão } \\
\text { integrativa }\end{array}$ & $\begin{array}{l}\text { Fazer uma revisão integrativa da } \\
\text { literatura, buscando evidências } \\
\text { científicas disponíveis sobre as ações } \\
\text { do profissional de enfermagem } \\
\text { durante o procedimento anestésico. }\end{array}$ & $\begin{array}{l}\text { Revista da Escola } \\
\text { de Enfermagem da } \\
\text { USP }\end{array}$ \\
\hline Sonobe et al., 2016 & $\begin{array}{l}\text { Assistência de enfermagem } \\
\text { perioperatória aos pacientes } \\
\text { com câncer de bexiga }\end{array}$ & $\begin{array}{l}\text { Caracterizar o perfil de pacientes } \\
\text { com câncer de bexiga em uma } \\
\text { unidade cirúrgica de um hospital } \\
\text { especializado em oncologia do } \\
\text { interior paulista e analisar os } \\
\text { cuidados de enfermagem } \\
\text { realizados para esses pacientes. }\end{array}$ & $\begin{array}{l}\text { Avances en } \\
\text { Enfermería }\end{array}$ \\
\hline Sobral et al., 2020 & $\begin{array}{l}\text { Atribuições do enfermeiro no } \\
\text { centro cirúrgico }\end{array}$ & $\begin{array}{l}\text { Discutir a execução destas atribuições } \\
\text { por parte do profissional. }\end{array}$ & Enfermagem Brasil \\
\hline $\begin{array}{l}\text { Souza, Palazzo } \\
\text { Montezallo, } 2020\end{array}$ & $\begin{array}{l}\text { Conhecimento dos profissionais de } \\
\text { enfermagem de centro cirúrgico } \\
\text { sobre hipotermia em pacientes } \\
\text { cirúrgicos oncológicos. }\end{array}$ & $\begin{array}{l}\text { Analisar o conhecimento dos } \\
\text { profissionais de enfermagem de } \\
\text { centro cirúrgico sobre hipotermia em } \\
\text { pacientes cirúrgicos oncológicos. }\end{array}$ & Revista SOBECC \\
\hline Souza et al., 2019 & $\begin{array}{l}\text { Percepção do cliente no } \\
\text { perioperatório sobre o cuidado de } \\
\text { enfermagem no centro cirúrgico. }\end{array}$ & $\begin{array}{l}\text { Analisar a percepção do cliente do } \\
\text { pré-operatório imediato e do } \\
\text { transoperatório sobre os cuidados de } \\
\text { enfermagem no centro cirúrgico. }\end{array}$ & $\begin{array}{l}\text { Revista Eletrônica } \\
\text { Acervo Saúde. }\end{array}$ \\
\hline
\end{tabular}




\begin{tabular}{|c|c|c|c|}
\hline Passos, 2012 & $\begin{array}{l}\mathrm{O} \text { cuidado da enfermagem ao } \\
\text { paciente cirúrgico frente ao ato } \\
\text { anestésico. }\end{array}$ & $\begin{array}{lrr}\text { Descrever as } & \text { responsabilidades } & \text { da } \\
\text { enfermagem da unidade } & \text { de } \\
\text { recuperação pós-anestésica } & \text { na } \\
\text { prevenção de complicações } & \text { pós- } \\
\text { operatórias imediatas. } & \end{array}$ & $\begin{array}{lr}\text { Perspectivas } & \text { on } \\
\text { line, ciências } \\
\text { biológicas e da } \\
\text { saúde. }\end{array}$ \\
\hline $\begin{array}{l}\text { Sillero-Sillero } \\
\text { Zabalegui, } 2019\end{array}$ & $\begin{array}{lcc}\text { Segurança } \quad \text { e } & \text { satisfação } & \text { de } \\
\text { pacientes com os cuidados } & \text { de } \\
\text { enfermeiros no perioperatório. } & \end{array}$ & $\begin{array}{l}\text { investigar a segurança e a satisfação } \\
\text { de pacientes e sua relação com os } \\
\text { cuidados dos enfermeiros no } \\
\text { perioperatório. }\end{array}$ & $\begin{array}{l}\text { Revista. Latino- } \\
\text { Americana } \\
\text { Enfermagem. }\end{array}$ \\
\hline Oliveira et al., 2020 & $\begin{array}{l}\text { Sistematização da assistência de } \\
\text { enfermagem no perioperatório de } \\
\text { ressecção transuretral de bexiga: } \\
\text { um relato de caso. }\end{array}$ & $\begin{array}{llll}\text { Relatar a implementação } & \text { da } \\
\text { sistematização da assistência } & \text { de } \\
\text { enfermagem perioperatória a um } \\
\text { paciente portador de câncer de } \\
\text { bexiga. }\end{array}$ & $\begin{array}{l}\text { Brazilian Journal of } \\
\text { Development. }\end{array}$ \\
\hline Niero et al., 2018 & $\begin{array}{l}\text { Cuidados de enfermagem ao } \\
\text { paciente oncológico em pós- } \\
\text { operatório } \\
\text { de cirurgia de cabeça e pescoço. }\end{array}$ & 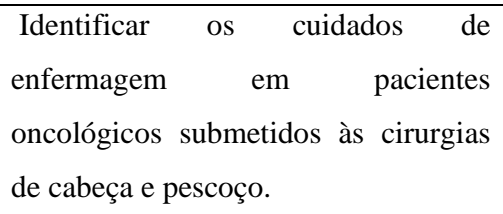 & RIES. \\
\hline Silva et al., 2019 & $\begin{array}{l}\text { Cuidados de enfermagem a } \\
\text { pacientes oncológicos: revisão } \\
\text { integrativa. }\end{array}$ & $\begin{array}{l}\text { identificar os cuidados de } \\
\text { enfermagem à pacientes oncológicos. }\end{array}$ & $\begin{array}{l}\text { Research, Society } \\
\text { and Development }\end{array}$ \\
\hline $\begin{array}{l}\text { Souza, Gonçalves \& } \\
\text { Alvarez, } 2018\end{array}$ & $\begin{array}{lcrr}\text { Cuidados } & \text { de } & \text { enfermagem } & \text { no } \\
\text { período } & \text { intraoperatório } & \text { para } \\
\text { manutenção } & \text { da } & \text { temperatura } \\
\text { corporal } & & & \\
& & \end{array}$ & $\begin{array}{llcr}\text { Descrever } & \text { os } & \text { cuidados } & \text { de } \\
\text { enfermagem para manutenção } & \text { da } \\
\text { temperatura corporal durante } & \text { o } \\
\text { intraoperatório. } & & \end{array}$ & Revista SOBECC \\
\hline Lima et al., 2019 & $\begin{array}{l}\text { Cuidados de enfermagem no } \\
\text { perioperatório de Transplante de } \\
\text { Células-Tronco Hematopoiéticas }\end{array}$ & $\begin{array}{l}\text { Analisar a produção científica sobre } \\
\text { os cuidados de enfermagem no } \\
\text { perioperatório dos Transplantes de } \\
\text { células-tronco hematopoiéticas. }\end{array}$ & $\begin{array}{l}\text { Revista Eletrônica } \\
\text { Acervo Saúde }\end{array}$ \\
\hline Soares, 2019 & $\begin{array}{l}\text { Diagnósticos de enfermagem no } \\
\text { pós-operatório de penectomia: } \\
\text { revisão integrativa }\end{array}$ & $\begin{array}{l}\text { Analisar as evidências científicas } \\
\text { produzidas na língua portuguesa e } \\
\text { espanhola em relação aos } \\
\text { diagnósticos de Enfermagem no pós- } \\
\text { operatório de penectomia. }\end{array}$ & Revista UNINGÁ \\
\hline $\begin{array}{l}\text { Ribeiro, Bonfim } \\
\text { Silveira, } 2011\end{array}$ & $\begin{array}{l}\text { Estratégias de capacitação da } \\
\text { equipe de enfermagem de um } \\
\text { centro cirúrgico oncológico }\end{array}$ & $\begin{array}{l}\text { Relatar a experiência da educação } \\
\text { Continuada aplicada à equipe de } \\
\text { enfermagem de um Centro Cirúrgico } \\
\text { de instituição de grande porte. } \\
\text { especializada em oncologia. }\end{array}$ & Revista SOBECC \\
\hline $\begin{array}{l}\text { Souza, Gonçalves \& } \\
\text { Alvarez, } 2018\end{array}$ & $\begin{array}{lcrr}\text { Cuidados } & \text { de } & \text { enfermagem } & \text { no } \\
\text { período } & \text { intraoperatório } & \text { para } \\
\text { manutenção } & \text { da } & \text { temperatura } \\
\text { corporal } & & & \\
& & \end{array}$ & $\begin{array}{lllr}\text { Descrever } & \text { os } & \text { cuidados } & \text { de } \\
\text { enfermagem } & \text { para manutenção } & \text { da } \\
\text { temperatura corporal durante } & \text { o } \\
\text { intraoperatório. } & & \end{array}$ & Revista SOBECC \\
\hline
\end{tabular}




\begin{tabular}{|c|c|c|c|}
\hline koch et al., 2018 & $\begin{array}{l}\text { Momento anestésico-cirúrgico: } \\
\text { transitando entre o conhecimento } \\
\text { dos(as) enfermeiros(as) e o } \\
\text { cuidado de enfermagem }\end{array}$ & $\begin{array}{l}\text { Verificar o conhecimento dos } \\
\text { enfermeiros(as) de um hospital } \\
\text { público da região oeste do estado de } \\
\text { Santa Catarina sobre o cuidado de } \\
\text { enfermagem no momento anestésico- } \\
\text { cirúrgico. }\end{array}$ & Revista SOBECC \\
\hline
\end{tabular}

Fonte: Autores (2021).

\section{Discussão}

\section{Categoria A - Riscos associados à anestesia no paciente oncológico}

Uma das complicações da anestesia geral em pacientes oncológicos são o desenvolvimento de paralisia das cordas vocais após a intubação. Frente aos fatores ligados ao paciente, observou-se que a incidência desses fatores aumenta à medida que os pacientes envelhecem. Em uma análise com múltiplas variações, constatou-se que o risco de pessoas acima de 50 anos ficou três vezes maior. Os registros realizados pela equipe médica também são muito importantes, pois mostraram que pessoas com diabetes e hipertensão também são mais susceptíveis a essa complicação. Além disso, aponta-se que a morbidade aumenta com o tempo de anestesia. Se a duração ultrapassar 6 horas, o risco é particularmente alto (Nazal et al., 2018).

Conforme Valdés, Garcia e Martínez (2019), o paciente que irá ser submetido a anestesia geral tem que permanecer em jejum para evitar a broncoaspiração e regurgitação, porém quando o paciente oncológico é privado de ingerir qualquer alimento por longos períodos, ocorrem diversas complicações, como: risco de desequilíbrio eletrolítico, hipoglicemia, ansiedade, hipotensão, diarreia e náusea. Para combater os problemas, a oferta de carboidratos, tais como malto dextrina, pode melhorar o controle metabólico interno (Pinto, Grigoletti \& Marcadenti, 2014).

$\mathrm{Na}$ anestesia geral de pacientes oncológicos, algumas complicações podem ser observadas, tais como: edema pulmonar, atelectasia, pneumonia, obstrução da VAS (vias aéreas superiores), broncoespasmo, edema de VAS, bronquite e exacerbar DPOC (Doença Pulmonar Obstrutiva Crônica), no qual aumenta o risco de infecção junto com o tempo de internação e retarda a cicatrização da ferida operatória, as complicações supracitadas podem ocorrer em até 30 dias do pósoperatório (Paiva et al., 2014).

Segundo Valdés, Garcia e Martínez (2019), os inalantes utilizados na anestesia geral produzem sobrecarga de fator indutível por hipóxia (HIF), que os tumores usam para sobreviver em condições de hipóxia e estão localizados no centro da massa tumoral para promover a proliferação e migração celular. Isso significa que o efeito dos agentes inalatórios é maior no que diz respeito ao impacto sobre o sistema imunológico dos pacientes. O propofol reduz a resposta imunológica adversa induzida pelo procedimento cirúrgico e a atividade antitumoral associada à inibição da ciclooxigenase, inibindo assim a prostaglandina E2.

Conforme Muñoz e Escobar (2019), o propofol não inibe a atividade antitumoral natural killer (NK), estando associado a uma menor incidência de metástases em tumores pulmonares experimentais. Outros autores propuseram que o fraco mecanismo antagonista $\beta$-adrenérgico do propofol está envolvido na proteção antitumoral do organismo dos pacientes. A cetamina é também outra opção de agente anestésico que fornece analgesia adequada ao paciente com o mínimo de efeitos colaterais sobre o sistema imunológico, por ter concentração plasmática reduzida. O contrário ocorre com os agentes opioides, que estão relacionados à sua capacidade de interferir na integridade da barreira hematoencefálica, seus potenciais efeitos de angiogênese nas células tumorais e seus efeitos imunossupressores diretos. É uma classe de fármacos controversa, que tem benefícios, porém são muitos os efeitos adversos, efeitos esses que podem alterar significativamente a trajetória de saúde de um paciente (Antunes et al., 2017). 
Rangel, Simões e Auler (2020), citam que durante a anestesia de pacientes oncológicos o uso de Betabloqueadores pode acelerar a multiplicação de células cancerígenas em todos os órgãos do corpo, pois os receptores beta-adrenérgicos frequentemente são encontrados nas células tumorais e com isto OU aumenta a disseminação de células tumorais.

Segundo Kearsley, Egan e McCaul (2018), o uso dos opioides no período perioperatório pode acarretar uma variedade de eventos adversos, que podem ser observados principalmente no pós-operatório, especialmente nas primeiras horas. A sedação prolongada pode levar ao despertar tardio do paciente e, às vezes, pode ser necessário administrar seu antagonista específico, que pode reverter o efeito analgésico residual. Da mesma forma, fraqueza da musculatura faríngea (que é um efeito colateral pouco conhecido) e depressão respiratória, são efeitos que exigem monitoramento durante a recuperação após a anestesia. Outros efeitos adversos são pruridos pós-operatório, náuseas e vômitos, retenção de urina, diminuição da motilidade gastrointestinal e obstrução intestinal paralítica. Esse grupo de drogas possui propriedades imunossupressoras e próangiogênicas, que podem aumentar o potencial metastático e a taxa de recorrência do câncer de pacientes submetidos à cirurgia tumoral para fins de tratamento. Atrasos na alta da unidade de recuperação pós-anestésica (URPA), aumento nas taxas de readmissão e hospitalização, bem como aumento nas complicações e morbidade perioperatória são consequências possíveis acerca do uso dessas drogas. (Garcia et al., 2020).

Conforme Alves e Faria (2014), uso de anestésicos opioide em pacientes oncológicos o uso de anestésicos opioides, como a morfina pode favorecer crescimento de algumas linhagens neoplásicas, por meio da ativação do receptor de crescimento epidérmico, dissipando o crescimento de tumores, como exemplo o tumor maligno de pulmão. O Fentanil, Remifentanil e sulfentanil utilizados em doses elevadas ocasionam a imunossupressão e alteram o funcionamento das células NK. Um dos opioides indicados para o controle da dor é a Petidina, pois não altera a função das células NK. Outro opioide que pode ser utilizado é o Tramadol, devido ao menor efeito supressor.

Para Lin, Gill e Kumar (2019), o paciente oncológico que utiliza grande quantidade de analgésico opioides pode piorar o prognóstico e aumentar o tempo de recuperação e desenvolver diversas comorbidades, como: íleo paralitico, retenção urinária e diminuição do nível de consciência. Yamada et al. (2019) afirma que o paciente oncológico durante a anestesia tem altos risco de apresentar sintomas compatíveis com náuseas e vômitos no período perioperatório, aumentando as chances de broncoaspiração, levando o paciente a adquirir uma pneumonite química.

Guzmán e López (2019), afirmam que um grande risco associado ao uso de opioide nos pacientes oncológicos é a síndrome de abstinência, ocasionada pelo uso em excesso de opioide durante a anestesia. Para combater a superdosagem de opioide é recomendado utilizar a bomba de PCA (Patient Controlled Analgesia), junto com medicamentos de ação coadjuvantes como Paracetamol, Gabapentina, Amitriptilina, Pregabalina e Clonidina. Para melhorar o controle álgico do paciente também e indicado o uso de anestesia local (Portella \& Stumm. 2016).

Valdés, Garcia e Martínez (2019), os anestésicos locais em geral apresentam uma característica importante em relação aos outros agentes, característica essa que que torna o uso dos agentes locais cada vez mais comum no controle da dor (dependendo do paciente e características tumorais, entre outros fatores): preservação da função das células NK. O resultado é a diminuição da proliferação de células tumorais, com consequente redução da possibilidade de recidiva do câncer.

A anestesia local, pode-se observar que o tecido tumescido aumento de volume, que apresenta pouco ou nenhum sangramento visível na incisão cirúrgica, apresenta-se em forma de gel e promove a exsudação do tecido durante a operação, reduzindo o inchaço, encurta o tempo de operação e reduz a exposição do paciente aos anestésicos inalados, bem como o uso em larga escala de opioides (Munhoz et al., 2020)

Para Brasil et al. (2018) anestesia de bloqueio de nervos periféricos em pacientes oncológicos pode apresentar complicações como: fadiga, constipação, comprometimento respiratório e prejuízos cognitivos. O bloqueio de nervos periférico é um procedimento difícil devido os pacientes oncológicos apresentarem alterações anatômicas ocasionadas pelo uso 
de quimioterapia e radioterapia.

\section{Categoria B - Os cuidados de enfermagem durante anestesia no paciente oncológico}

A atuação do enfermeiro nos procedimentos anestésicos é fundamental para o planejamento e organização de materiais e equipamentos, cooperação com o anestesiologista e acompanhamento do paciente após a anestesia, não sendo o apoio da equipe de enfermagem dependente apenas da interação do enfermeiro e do anestesiologista como auxiliares, devendo estes atuar com conhecimentos científicos e orientações para prestar uma assistência de forma eficaz e com qualidade, que favoreça a segurança do paciente. Ao estabelecer um plano de enfermagem para definir a atuação do enfermeiro frente aos procedimentos de anestesia, ele irá nortear e auxiliar no planejamento e mostrar a importância do profissional em centro cirúrgico (Lemos, Peniche. 2016).

O principal cuidado do enfermeiro antes da indução anestésica do paciente oncológico é realizar o check-list, pois antes o enfermeiro deve conferir todos os dados do paciente e ter certeza que a equipe multiprofissional averiguou os equipamentos, como o teste de monitores, laringoscópios e o carrinho de anestesia, prover o material e medicação essencial para o início da anestesia, averiguar a disponibilidade de Unidade de terapia intensiva (UTI), hemocomponentes e antibióticos, além de coletar informações como alergias e jejum (Koch et al., 2018).

$\mathrm{O}$ enfermeiro deve promover um ambiente seguro e propício para a anestesia e realizar uma assistência de enfermagem segura para ter um procedimento cirúrgico tranquilo. Os pacientes antes de ser submetido a anestesia devem passar por um preparo realizado nas unidades de internação, contemplando minimamente 8 horas de jejum antes do procedimento cirúrgico. Às vezes devido ao atraso do centro cirúrgico, o tempo de jejum pode se prolongar e ocasionar várias alterações fisiológicas e hemodinâmicas nos pacientes oncológicos. O enfermeiro que está na assistência do bloco cirúrgico deve sinalizar as unidades de internação para a oferta de maltodextrina ou outro complemento alimentar, que deve ser administrado duas horas antes da cirurgia para evitar broncoaspiração e regurgitamento durante a anestesia e prevenir possíveis casos de desnutrição e alterações fisiológicas (Souza et al., 2019)

Os pacientes oncológicos podem desenvolver hipotermia durante a indução anestésica devido a sua perca de massa muscular e desnutrição ocasionada pelo uso de quimioterápicos, radioterápicos e tumoração. $\mathrm{O}$ uso de anestésicos durante o procedimento cirúrgico, facilita que haja uma queda da temperatura corporal, no qual necessita o monitoramento assíduo dos sinais vitais pela equipe de enfermagem. Alguns cuidados de enfermagem que podem ser acrescentados durante o período anestésico para que a temperatura corporal do paciente seja controlada e não ocorra a hipotermia são a infusão de solução aquecida, manta e colchão térmico, umidificação e aquecimento dos gases administrados (Souza, Gonçalves \& Alvarez, 2018).

Para realizar o controle da hipotermia, o enfermeiro deve ter como instrumento norteador a Sistematização da assistência de enfermagem perioperatória (SAEP), a fim de direcionar e operacionalizar os cuidados de enfermagem individual, integral, sistematizada e documentada. Por meio da SAEP, o enfermeiro deve traçar um plano de enfermagem seguro no qual zele pela integridade do paciente durante a indução anestésica (Soares, 2019).

Conforme Lima et al. (2019) e Oliveira et al. (2020), os principais diagnósticos de enfermagem para o paciente oncológico submetido a anestesia são: risco de broncoaspiração, risco de queda, ansiedade, nutrição desiquilibrada conforto prejudicado, desobstrução ineficaz das vias aéreas, risco de integridade da pele prejudicada, risco de lesão na córnea, risco de lesão por posicionamento perioperatório, hipotermia e desiquilíbrio hidroeletrolítico.

Conforme Santana, Souza e Viana (2018), as intervenções de enfermagem no paciente oncológico submetido a anestesia têm a finalidade de prevenir e minimizar as possíveis complicações durante o procedimento de indução anestésica na qual tem o papel fundamental na segurança do paciente, as intervenções indicadas são: restabelecer o equilíbrio hidroeletrolítico do paciente, monitoramento dos sinais vitais (principalmente o controle da temperatura corporal), manter 
monitoramento contínuo, verificar sinais de hipóxia, testar todos os equipamentos anestésicos, observar indícios de choque hipovolêmico e prevenir lesão por posicionamento cirúrgico. É função do enfermeiro elaborar um plano assistencial individualizado para fornecer os cuidados de enfermagem essenciais ligados aos diagnósticos e intervenções traçadas pelo enfermeiro (Santos et al., 2017).

Os cuidados de enfermagem fundamentais durante a indução anestésica são a manutenção da posição adequada do paciente na mesa cirúrgica com exposição da via aérea superior, monitorização do paciente, teste dos aparelhos, aspirador de secreção montado e próximo do anestesista, acompanhar e indução anestésica e nunca deixar o anestesista sozinho na sala cirúrgica, preservar a integridade física do paciente e mantê-lo aquecido, em caso de procedimentos invasivos como passagem de cateter venoso central (CVC) e cateteres de monitorização invasiva a equipe de enfermagem deve prover material e auxiliar o anestesista durante o procedimento (Torres et al., 2020)

Finalizada a anestesia, a equipe de enfermagem deve prestar os seguintes cuidados: auxiliar o anestesista no processo e extubação, deixar VAS pérvia, ofertar oxigênio, monitorizar sinais vitais, manter o paciente sempre com o cinto de segurança, em posição supina a $30^{\circ}$ e aquecê-lo com manta e colchão térmico para prevenir a hipotermia e avaliar o nível de sedação com o uso das escalas RAMSAY e RASS. O enfermeiro deve estar sempre atento a sinais de náusea e vômito para o paciente não broncoaspirar (Sonobe et al., 2016).

Conforme Passos (2012), para o paciente ser encaminhado para a recuperação anestésica o enfermeiro deve avaliar o nível de consciência do paciente, respiração e reflexos de tosse, sinais vitais estáveis, dor controlada, volemia adequada e controle de hemorragias, além de avaliar se durante a indução anestésica, as ações de enfermagem implementadas foram eficazes.

Segundo Silva et al. (2019) os pacientes oncológicos submetidos a anestesias precisam dos cuidados de enfermagem humanizados, pois os enfermos apresentam ansiedade, medo e angústia, o acolhimento da família, por meio de um plano de cuidado direcionado e pautado para a humanização, no qual vai refletir no estado fisiológico do paciente durante a indução anestésica.

Para Niero et al. (2018), além do controle emocional o enfermeiro deve realizar a assistência individualizada para o controle álgico, pois as cirurgias oncológicas em sua grande parte são bem agressivas, no qual o paciente apresenta comprometimento de vários órgãos. Os pacientes oncológicos submetidos anestesias podem apresentar dor intensa devido a manipulação, assim o enfermeiro deve aplicar escalas de dor e realizar avaliação para a infusão de analgésicos opioides com a finalidade de reduzir e aliviar a dor do paciente.

Conforme Sillero-Sillero e Zabalegui (2019), os cuidados de enfermagem com efeitos anestésicos após procedimento cirúrgico tem como objetivo manter os parâmetros de sinais vitais estáveis, equilíbrio hemodinâmico e controle da dor, detectar e prevenir eventos adversos, minimizando qualquer complicação. Com isto, o enfermeiro deve ter conhecimento e habilidade para gerenciar o plano assistencial durante a anestesia do paciente oncológico

Segundo Ribeiro, Bonfim e Silveira (2011), no centro cirúrgico, o enfermeiro necessita ter conhecimento das técnicas necessárias para que o paciente seja tratado da melhor forma possível e para que não haja complicações futuras. O tratamento oncológico cirúrgico requer um preparo específico da equipe de enfermagem, pois lida com paciente em particulares situações e problemas. Portanto, é de extrema importância que a enfermagem desenvolva diariamente o aperfeiçoamento das técnicas e cuidados prestados ao paciente nesse tipo de procedimento, para que assim, os riscos sejam diminuídos e a haja uma recuperação rápida do paciente. 


\section{Considerações Finais}

As complicações da anestésia geral em pacientes oncológicos são o desenvolvimento de paralisia das cordas vocais após a intubação, o tempo prologado de jejum, edema pulmonar, atelectasia, pneumonia, obstrução da VAS, broncoespasmo, edema de VAS, bronquite e exacerbar DPOC.

Os anestésicos utilizados podem gerar complicações, como a resposta imunológica adversa induzida pelo procedimento cirúrgico e a atividade antitumoral associada à inibição da ciclooxigenase, inibindo assim a prostaglandina E2. Estudos em animais demonstraram que, em comparação com outros anestésicos e interferir na integridade da barreira hematoencefálica, seus potenciais efeitos de angiogênese nas células tumorais e seus efeitos imunossupressores diretos, além de acelerar a multiplicação de células cancerígenas em todo o organismo.

A sedação prolongada pode levar ao despertar tardio do paciente e, às vezes, ocasionar fraqueza da musculatura faríngea, depressão respiratória, pruridos, náuseas, vômitos, retenção de urina, diminuição da motilidade gastrointestinal e obstrução intestinal paralítica.

A atuação do enfermeiro nos procedimentos anestésicos é fundamental para promover um ambiente seguro e propicio para a anestesia, além de uma assistência de enfermagem segura. $\mathrm{O}$ enfermeiro deve prever e prover os materiais cirúrgicos para o paciente e atuar diretamente no cuidado ao paciente. Um dos principais cuidados de enfermagem é a segurança do paciente, no qual o enfermeiro deve sempre checar o nome do paciente, aplicação dos termos e avaliações realizadas no momento pré-operatório. O paciente deve sempre estar em jejum de 8 horas e atentar-se para alergias e patologias de base.

É importante que toda a equipe cheque os equipamentos antes da indução anestésica para passar segurança e evitar estresse, o principal instrumento utilizado neste momento é o checklist de cirurgia segura, no qual o anestesista vai testar todo o carrinho de anestesia, monitores e laringoscópio, além de prover toda a medicação e materiais necessários para indução anestésica.

A equipe de enfermagem antes da indução deve monitorar o paciente e deixar coxins de posicionamento prontos para serem utilizados para expor a VAS do paciente, além de manter o paciente aquecido e coberto durante toda a indução anestésica, e deixa o aspirador de VAS sempre testado e pronto para o uso em caso de vomito. O paciente oncológico apresenta diversas comorbidades e para prestar a assistência segura o enfermeiro deve ter o conhecimento de cada etapa do período perioperatório para prevenir possíveis complicações durante toda anestesia.

Durante a pesquisa Foi notado que na literatura temos uma escassez de publicação na área de cuidados de enfermagem em pacientes oncológicos submetido à anestesia, pois hoje a enfermagem foca muito nos cuidados paliativos, esperamos que o trabalho realizado possa contribuir para o desenvolvimento futuro de novos trabalhos que possam abordar os pacientes oncológicos submetidos a anestesia.

\section{Referências}

Antunes, M. M et al. (2017). Recorrência do câncer: anestesia é realmente vilã? Revista Médica Minas Gerais. 27(2), S97-S105. 10.5935/22383182.20170022 .

Alves, D. R. \& Faria, M. (2014). Anestesia e Recidiva Oncológica - Será tempo de agir? Revista Sociedade Portuguesa de Anestesiologia. 23(4). https://doi.org/10.25751/rspa.6233.

Brasil, E. A et al. (2018). Métodos anestésicos intervencionistas no tratamento da dor oncológica. Acta médica. 39(2): 202-213.

Cesário, J. M. S.; Flauzino, V. H. P. \& Mejia, J. V. C. (2020). Metodologia científica: Principais tipos de pesquisas e suas caraterísticas. Revista Científica Multidisciplinar Núcleo do Conhecimento, 5(11), 23-33. 10.32749/nucleodoconhecimento.com.br/educacao/tipos-de-pesquisas.

Garcia, O. L et al. (2020) Ventajas de la anestesia intravenosa libre de opioides en cirugía ambulatoria oncológica de mama. Revista Ciencias Médicas. 24(5): e4648.

Guzmán, F. C. \& López, G. F. (2019). Analgesia perioperatoria en el paciente oncológico con uso crónico de opioides. Revista Mexicana de Anestesiología, 42(3), $187-190$ 
Kearsley, R.; Egan, S. \& Mccaul, C. (2018). Anestesia para cirurgia citorredutora (CRS) com quimioterapia intraperitoneal hipertérmica (HIPEC). Word Ffederantin of Societes of anaesthesiologists. 379, 1-7.

Koch, T. M et al. (2018). Momento anestésico-cirúrgico: transitando entre o conhecimento dos(as) enfermeiros(as) e o cuidado de enfermagem. Revista SOBECC. 23(1): 7-13. 10.5327/Z1414-4425201800010003.

Lemos, C. S. \& Peniche, A. C. G. (2016). Assistência de enfermagem no procedimento anestésico: revisão integrativa. Revista Escola de Enfermagem USP. 50(1):158-166. http://dx.doi.org/10.1590/S0080-623420160000100020.

Lima, F. C et al. (2019). Cuidados de enfermagem no perioperatório de Transplante de Células-Tronco Hematopoiéticas. Revista Eletrônica Acervo Saúde. Vol. Sup.36, e1702, https://doi.org/10.25248/reas.e1702.2019.

Lin, C.; Gill, R. \& Kumar, K. (2019). Bloqueio bilateral do plano eretor da espinha torácica inferior em cirurgia oncológica ginecológica aberta por via abdominal: série de casos. Revista da Sociedade Portuguesa de Anestesiologia. 23(4), 113-122. https://doi.org/10.25751/rspa.6233.

Munhoz, C et al. (2020). Uso de anestesia tumescente em mastectomia radical unilateral relato de caso. Anais do Salão Internacional de Ensino, Pesquisa e Extensão, 8(1), 14.

Muños, S. G. \& Escobar, I. C. (2019). Anestesia total intravenosa en cirugia oncológica de mama. Revista Cubana de Anestesiología y Reanimación. 18(2):e494.

Nazal, C. H et al. (2021). Paralisia de cordas vocais após intubação endotraqueal: uma complicação incomum da anestesia geral. Revista Brasileira de Anestesiologia. 637-640. http://dx.doi.org/10.1016/j.bjane.2017.12.007.

Oliveira, P. C. D, et al. (2020). Sistematização da assistência de enfermagem no perioperatório de ressecção transuretral de bexiga: um relato de caso. Brazilian Journal of Development. 6(11), 92823-92832. 10.34117/bjdv6n11-624.

Paiva, T. M et al. (2014). Complicações respiratórias em cirurgias oncológicas de grande porte em cabeça e pescoço: revisão de literatura. Revista Brasileira de Cirurgia de Cabeça Pescoço, 43(4), 213-219.

Passos, A. P. P. (2012). O cuidado da enfermagem ao paciente cirúrgico frente ao ato anestésico. Perspectiva Online. v. 2 n. 6: Edição Comemorativa IV CICC. https://doi.org/10.25242/8868262012202.

Pinto, A. S.; Grigoletti, S. S. \& Marcadenti, A. (2015). Abreviação do jejum entre pacientes submetidos à cirurgia oncológica: revisão sistemática. Arquivos Brasileiros de Cirurgia Digestiva. 28(1):70-73. http://dx.doi.org/10.1590/S0102-67202015000100018.

Portella, M. P., \& Stumm, E. M. F. (2016). Intensidade da dor de pacientes em pós-operatório de cirurgia oncológica. XXIV Seminário de Iniciação Científica.

Rangel, F.; Simões, C. M. \& Auler, J. O. C. (2020). Anestesia no paciente oncológico: as técnicas e agentes anestésicos podem influenciar o desfecho destes pacientes? Uma revisão narrativa. Revista de Medicina. 99(1):40-5. http://dx.doi.org/10.11606/issn.1679-9836.v99i1p40-45.

Ribeiro, M. B.; Bonfim, I. M. \& Silveira, C. T. (2011). Estratégias de capacitação da equipe de enfermagem de um centro cirúrgico oncológico. Revista SOBECC. $163: 21-29$.

Santana, C. C. C.; Souza, J. R. S.; Viana, D. A. (2018). Análise das ações de enfermagem nas fases cirúrgicas da mastectomia: uma revisão sistemática. Revista Brasileira de Inovação Tecnológica em Saúde, 8(2). https://doi.org/10.18816/r-bits.v8i2.15556.

Santos, M. R et al. (2012). A importância da Assistência de Enfermagem na Sala de Recuperação Pós- Anestésica: Visão dos Monitores em Enfermagem Cirúrgica. International Nursing Congress Theme: Good practices of nursing representations In the construction of society,

Silva, F. S et al. (2019). Cuidados de enfermagem a pacientes oncológicos: Revisão integrativa. Research, Society and Development 8(6):e35861037. http://dx.doi.org/10.33448/rsd-v8i6.1037.

Silva, S. S. F et al. (2020). Pós-operatório de paciente oncológico em jejum prolongado: diagnósticos e intervenções de enfermagem. Revista enfermagem UERJ, 28:e50567. DOI: http://dx.doi.org/10.12957/reuerj.2020.50567.

Sillero-Sillero, A. \& Zabalegui, A. (2019). Segurança e satisfação de pacientes com os cuidados de enfermeiros no perioperatorio. Revista Latino-Americana de Enfermagem. 27:e3142. 10.1590/1518-8345.2646.3142.

Soares, D. F. S. (2019). Diagnósticos de enfermagem no pós-operatório de penectomia: revisão integrativa. Revista Uningá, 56(S6), 179-193.

Sobral, G. A. S et al. (2019). Atribuições do enfermeiro no centro cirúrgico. Enfermagem Brasil. 18(4):603-9. https://doi.org/10.33233/eb.v18i4.3117.

Sonobe, H. M et al. (2016). Assistência de enfermagem perioperatória aos pacientes com câncer de bexiga. Avances em Enfermagem 34(2):159-169. 10.15446/av.enferm.v34n2.37465.

Souza, A.; Palazzo, S. \& Montezello, D. (2017). Conhecimento dos profissionais de enfermagem de centro cirúrgico sobre hipotermia em pacientes cirúrgicos oncológicos. Revista. SOBECC. 22(4): 188-192. 10.5327/Z1414-4425201700040003.

Souza, I. B et al. (2017). Percepção do cliente no perioperatório sobre o cuidado de enfermagem no centro cirúrgico. Revista Eletrônica Acervo Saúde. Vol.Sup.26, e860, 7/2019. https://doi.org/10.25248/reas.e860.2019.

Souza, È, O.; Gonçalves, N. \& Alvarez, A. G. (2019) Cuidados de enfermagem no período intraoperatório para manutenção da temperatura corporal. Revista SOBECC. 24(1): 31-36. 10.5327/Z1414-4425201900010007. 
Research, Society and Development, v. 10, n. 5, e31310514798, 2021

(CC BY 4.0) | ISSN 2525-3409 | DOI: http://dx.doi.org/10.33448/rsd-v10i5.14798

Torres, R. L. N et al. (2020) Assistência de enfermagem perioperatória ao paciente submetido a laparotomia. Enfermagem: Inovação, Tecnologia e Educação em Saúde. 5(3): 100-110. 10.37885/200700773.

Valdés, M. E. O.; Garcia, O. L. \& Martínez, M J. F. (2019). Perioperatorio y recurrencia oncológica: reto actual en la práctica anestésica. Revista Cubana de Anestesiología y Reanimación. 19(1):e525.

Yamada, L. A. P et al. (2019). Desenvolvimento de um modelo preditivo multivariado para náusea e vômito no pós-operatório de cirurgia oncológica em adultos. Revista Brasileira de Anestesiologia. 69(4):342---349. https://doi.org/10.1016/j.bjan.2019.03.002. 